\title{
Povidona Yodada como Antiséptico Oral en la Reducción de la Carga Viral del SARS-CoV-2: Revisión de la Literatura
}

\author{
Povidone lodine as an Oral Antiseptic in the Reduction \\ of SARS-CoV-2 Viral Load: Literature Review
}

\author{
Andrés Anwandter ${ }^{1}$; Cristóbal Lefno Diocares²; Camila Valeria ${ }^{3} \&$ Diego Robledo $^{2}$
}

\begin{abstract}
ANWANDTER, A.; LEFNO-DIOCARES. C.; VALERIA, C. \& ROBLEDO, D. Povidona yodada como antiséptico oral en la reducción de la carga viral del SARS-CoV-2: Revisión de la literatura. Int. J. Odontostomat., 15(4):997-1004, 2021.

RESUMEN: Se ha sugerido que el uso de antisépticos orales podría reducir la carga viral del virus SARS-CoV-2 en los pacientes durante la atención dental, pero sin evidencia que avale su efectividad. Dada la vulnerabilidad del virus a la oxidación, se ha recomendado el uso de colutorios que contengan agentes oxidantes como la povidona yodada. El objetivo de la presente revisión fue determinar la efectividad del uso de povidona yodada como antiséptico oral en la disminución de la carga viral del virus SARS-CoV-2. Se realizó una búsqueda bibliográfica en PubMed, Biblioteca Virtual en Salud, SciELO, Web of Science y EBSCO host. Se incluyeron estudios clínicos en pacientes con COVID-19 y estudios in vitro con cepas del virus que utilizaran colutorios de povidona yodada como forma de intervención, publicados entre enero del 2019 y enero del 2021. La selección de los artículos se realizó en dos etapas por dos autores de manera independiente. Luego de eliminar los artículos duplicados, se mantuvieron 53 referencias. Finalmente se incluyeron 2 estudios in vivo y 5 estudios in vitro para la revisión cualitativa. En los estudios in vitro, todas las concentraciones de povidona yodada evidenciaron una actividad virucida eficaz en los distintos tiempos de exposición, donde la mínima concentración efectiva correspondió a 0,5\% en 15 segundos. Los estudios in vivo presentaron resultados positivos hacia el uso de povidona yodada, pero con tamaños muestrales pequeños y una gran heterogeneidad en su metodología. En conclusión el uso profiláctico de povidona yodada como colutorio contra el virus SARS-CoV2 es respaldado por los trabajos in vitro, con tiempos de aplicación fácilmente realizables en la atención dental, pero se requiere de un mayor número de ensayos controlados aleatorizados para comprobar su efectividad en la práctica clínica.
\end{abstract}

PALABRAS CLAVE: COVID-19, povidona yodada, antisépticos orales, profilaxis dental.

\section{INTRODUCCIÓN}

En diciembre del 2019, una serie de casos de infecciones respiratorias agudas sin etiología conocida se reportaron en la ciudad de Wuhan, China (Huang et al., 2020). Estos habían sido provocados por un nuevo patógeno viral del género Beta corona virus, denominado posteriormente como coronavirus de tipo 2 causante del síndrome respiratorio agudo severo (SARS-CoV-2) y responsable de la enfermedad de COVID-19 (Organización Mundial de la Salud, 2020a). En los meses posteriores, la propagación del virus se expandió fuera de China y el número de casos reportados con la enfermedad aumentó rápidamente, sien-

\footnotetext{
${ }^{1}$ Cirujano dentista, Puerto Montt, Chile.

${ }^{2}$ Escuela de Medicina, Universidad Austral de Chile, Valdivia, Chile.

${ }^{3}$ Cirujano dentista, Quemchi, Chiloé, Chile.
}

do reconocida como una pandemia el 11 de marzo del 2020 por la Organización Mundial de la Salud (2020b).

El virus puede transmitirse a través de gotitas durante el contacto cercano cara a cara con portadores asintomáticos, presintomáticos y sintomáticos (Wiersinga et al., 2020) y se ha detectado su presencia en la saliva del $91,7 \%$ de los pacientes que presentan la enfermedad (To et al., 2020). Esto resulta sumamente relevante en el entorno odontológico, donde la atención implica contacto cercano con los pacientes y en donde se ha reconocido el riesgo micro-

Received: 2021-06-09 Accepted: 2021-12-12 
biológico relacionado con la presencia de aerosoles y salpicaduras, representando una ruta potencial para la transmisión de enfermedades (Harrel \& Molinari, 2004).

Por esta razón, la reducción de la carga viral presente en la saliva de los pacientes se ha convertido en un importante objetivo durante los procedimientos de alto riesgo como lo son los tratamientos dentales. Para esto, se ha sugerido el uso de antisépticos orales por su potencial efecto sobre la envoltura lipídica del virus,que influiría en la replicación del virus durante etapas tempranas de la enfermedad (O'Donnell et al., 2020). La Asociación Dental Americana (ADA) ha sugerido que los pacientes utilicen enjuagues bucales de manera previa a su tratamiento para minimizar el riesgo de transmisión del virus, pero sin evidencia que avale estas recomendacione (American Dental Association, 2020). Dentro de los antisépticos propuestos por la ADA se encuentra la povidona yodada o yodo povidona al $0,2 \%$, la cual se ha planteado como una opción para inactivar el SARS-CoV-2 debido a la vulnerabilidad que tendría el virus frente a la oxidación (American Dental Association). Se ha reportado que las soluciones de povidona yodada han inactivado eficazmente una amplia gama de patógenos, en donde destacan virus como el SARS-CoV y el MERS, los cuales han sido inactivados en tiempos de contacto desde los 15 segundos (Eggers et al., 2018). Esto, sumado a su falta de resistencia microbiana (Kramer et al., 2018), la seguridad de su uso en la cavidad oral con concentraciones de hasta el 2,5\% (Frank et al., 2020), su bajo costo y amplia distribución en el mercado, han generado un gran interés por el uso de povidona yodada contra el SARS-CoV-2.

Durante los meses posteriores al primer brote de la enfermedad en la ciudad de Wuhan, se han reportado distintos trabajos tanto in vitro como in vivo que han intentado estudiar el efecto del uso de povidona yodada como colutorio sobre el virus causante de la enfermedad por COVID-19, para respaldar su uso como medida estándar en las estrategias de prevención dispuestas por los distintos organismos de salud. Al no existir revisiones actualizadas sobre estos estudios, la presente revisión busca determinar la efectividad del uso de povidona yodada como antiséptico oral en la disminución de la carga viral del virus SARS-CoV-2.

\section{MATERIAL Y MÉTODO}

Se realizó una búsqueda bibliográfica de la literatura en cinco bases de datos (PubMed, Biblioteca Virtual en Salud, SciELO, Web of Science y EBSCO host) el día 23 de febrero del 2021. Se definieron como criterios de inclusión estudios clínicos en pacientes con COVID-19 o estudios in vitro con cepas del virus SARSCoV-2 que utilizaran colutorios de povidona yodada como forma de intervención sobre la carga viral, publicados entre enero del 2019 y enero del 2021. Como criterios de exclusión se consideraron artículos que no contaran con un grupo control, artículos de revisión, cartas al editor, resúmenes de conferencias, reportes de casos y columnas de opinión. No se establecieron restricciones de idioma, género o edad de los participantes. Los términos utilizados en la búsqueda se detallan en la Tabla I.

Luego de la búsqueda, se removieron los artículos duplicados y se llevó a cabo la selección de los trabajos restantes en base a los criterios de inclusión establecidos. Esta fue realizada por dos de los investigadores (A.E.A.F. y C.R.L.D.) de manera independiente, quienes evaluaron los trabajos por título y resumen. Una vez efectuada la primera selección, se realizó la lectura completa de estos artículos por los mismos investigadores de manera independiente para realizar la selección definitiva. En caso de discrepancias en la selección, se designó un tercer investigador (C.A.V.V.) para participar de la decisión final. Se evaluó el nivel de acuerdo entre los investigadores en esta última etapa en base al índice de kappa.

Tabla I. Estrategia de búsqueda.

\begin{tabular}{|c|c|}
\hline PubMed & $\begin{array}{l}\left.\left.\text { ((mouthwash*) OR (mouthrinse*) OR (gargl }{ }^{*}\right)\right) \text { AND ((COVID-19) OR (SARS-COV-2)) AND ((Povidone- } \\
\text { lodine) OR (PVP-I) OR (PVP-lodine) OR (Polyvinylpyrrolidone-lodine)) }\end{array}$ \\
\hline DIVII & ((COVID-19) OR (SARS-COV-2)) AND ((Mouthrinse\$) OR (Mouthwash\$) OR (GargI\$)) AND \\
\hline & ((Povidone-lodine) OR (PVP-I) OR (PVP-lodine) OR (Polyvinylpyrrolidone-lodine)) \\
\hline SciELO & $\begin{array}{l}\text { ((COVID-19) OR (SARS-COV-2)) AND ((Mouthrinse*) OR (Mouthwash*) OR (Gargl*)) AND (("Povidone } \\
\text { lodine") OR ("PVP I") OR ("PVP lodine") OR ("Polyvinylpyrrolidone lodine")) }\end{array}$ \\
\hline Web of Science & $\begin{array}{l}\left.\left.\text { ALL }=\left(\left(\text { mouthwash }^{*}\right) \text { OR (mouthrinse }\right) \text { OR (gargl }{ }^{*}\right)\right) \text { AND ALL }=((\text { COVID-19) OR (SARS-COV-2) }) \text { AND } \\
\text { ALL }=((\text { Povidone-lodine) OR (PVP-I) OR (PVP-lodine) OR (Polyvinylpyrrolidone-lodine }))\end{array}$ \\
\hline BSCOhost & $\begin{array}{l}\text { (covid-19 or sars-cov-2 or coronavirus) and (garg|* or mouthwash* or mouthrinse* or "oral rinse") and } \\
\text { ("povidone iodine" or "PVP I" or "Polyvinylpyrrolidone lodine" or "PVP lodine") }\end{array}$ \\
\hline
\end{tabular}


Para evaluar el riesgo de sesgo en la metodología de los estudios in vivo, se utilizó la herramienta "Riesgo de sesgo" desarrollada por la "Cochrane Collaboration" (Higgins et al., 2011). Al no existir una herramienta estándar para evaluar el riesgo de sesgo en los estudios in vitro, esta se realizó en base a una revisión publicada anteriormente (Golbach et al., 2016) modificando algunos de sus parámetros de acuerdo con los criterios de elegibilidad de este estudio.

Posteriormente, se realizó una revisión cualitativa de la información a partir de los artículos seleccionados. Los datos faltantes en los estudios fueron consultados con sus respectivos autores.

\section{RESULTADOS}

A partir de la lectura del título y resumen, se identificaron 124 artículos en las cinco bases de datos (PubMed/Medline, Biblioteca Virtual en Salud, Scielo, Web of Science y EBSCO host) publicados entre Enero del 2019 y Enero del 2021 (Fig. 1).
Luego de remover los duplicados (71), se mantuvieron 53 artículos. Posteriormente, se realizó la lectura del título y resumen, donde se aplicaron los criterios de selección y 43 trabajos fueron excluidos, resultando en 10 trabajos seleccionados. Luego de la lectura completa y selección final, 3 artículos fueron excluidos (Tabla II), resultando en 7 trabajos para la síntesis cualitativa. Se calculó el índice kappa para evaluar el nivel de acuerdo entre los investigadores en la selección final de los artículos, este correspondió a 1, lo que indicó un grado de acuerdo perfecto (100\%).

Tabla II. Artículos excluidos y causa de exclusión $(n=3)$.

\begin{tabular}{ccc}
\hline$N^{\circ}$ & \multicolumn{1}{c}{ Autor } & \multicolumn{1}{c}{ Causa de exclusión } \\
\hline 1 & Jain et al. & Ausencia de grupo control negativo \\
2 & Martínez et al. & Ausencia de grupo control negativo \\
3 & Statkute et al. & Ausencia de grupo control negativo
\end{tabular}

1. Chlorhexidine: An effective anticovid mouth rinse.

2. Is povidone iodine mouthwash effective against SARS-CoV-2? First in vivo tests.

3. Brief Report: The Virucidal Efficacy of Oral Rinse Components Against SARS-CoV-2 In vitro.

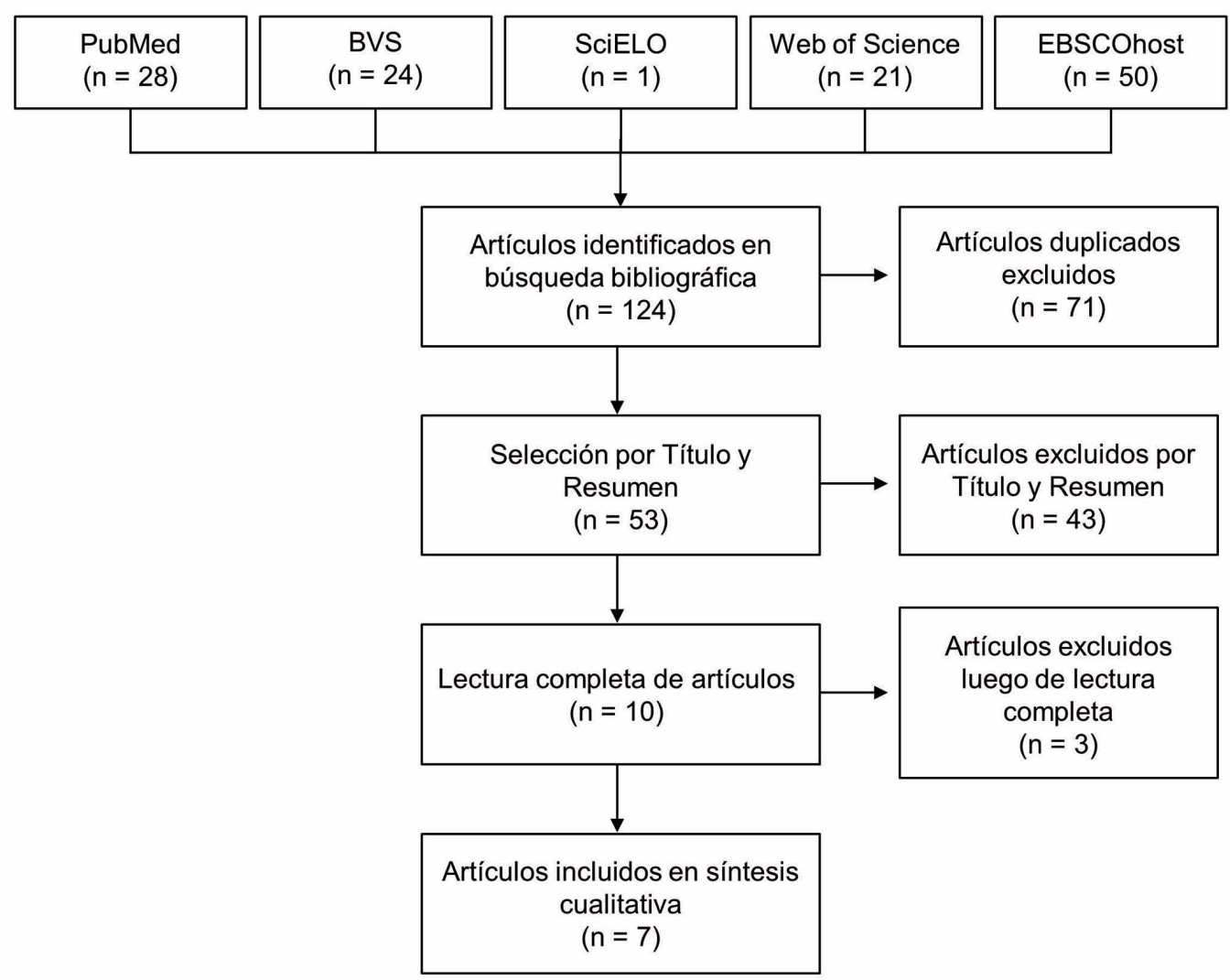

Fig. 1. Diagrama de flujo del proceso de selección de artículos. BVS: Biblioteca Virtual en Salud. 
De los artículos incluidos, 5 correspondieron a estudios in vitro (Anderson et al., 2020; Bidra et al., 2020a,b; Hassandarvish et al., 2020; Pelletier et al., 2020) y 2 correspondieron a estudios in vivo (Mohamed et al., 2020; Seneviratne et al., 2020), publicados entre junio del 2020 y diciembre del 2020. Las concentraciones de povidona yodada utilizadas en los estudios in vitro correspondieron a $0,5 \%, 0,75 \%, 1 \%, 1,25 \%$, y $1,5 \%$, mientras que en los estudios in vivo se utilizaron concentraciones de $0,5 \%$ y $1 \%$.

Riesgo de sesgo en los estudios. Al realizar la evaluación del riesgo de sesgo en los estudios in vitro, esta se basó en un revisión publicada anteriormente (Golbach et al.) y se modificaron algunos de los parámetros de acuerdo con los criterios de elegibilidad de este estudio (Tabla III). Solo un parámetro evaluado fue considerado de "Alto riesgo" en todos los artículos, correspondiente al patrocinio recibido para la realización de las investigaciones.

Para evaluar el riesgo de sesgo en los estudios in vivo, se utilizó la herramienta "Riesgo de sesgo" desarrollada por la "Cochrane Collaboration" (Higgins et al.) (Tabla IV).

Mohamed et al. presentó un alto riesgo de sesgo en el ocultamiento de la asignación, y en el cegamiento de participantes y personal de la investigación. Seneviratne et al., no presentó ningún parámetro de alto riesgo.

Tabla III. Riesgo de sesgo en los estudios in vitro $(n=5)$

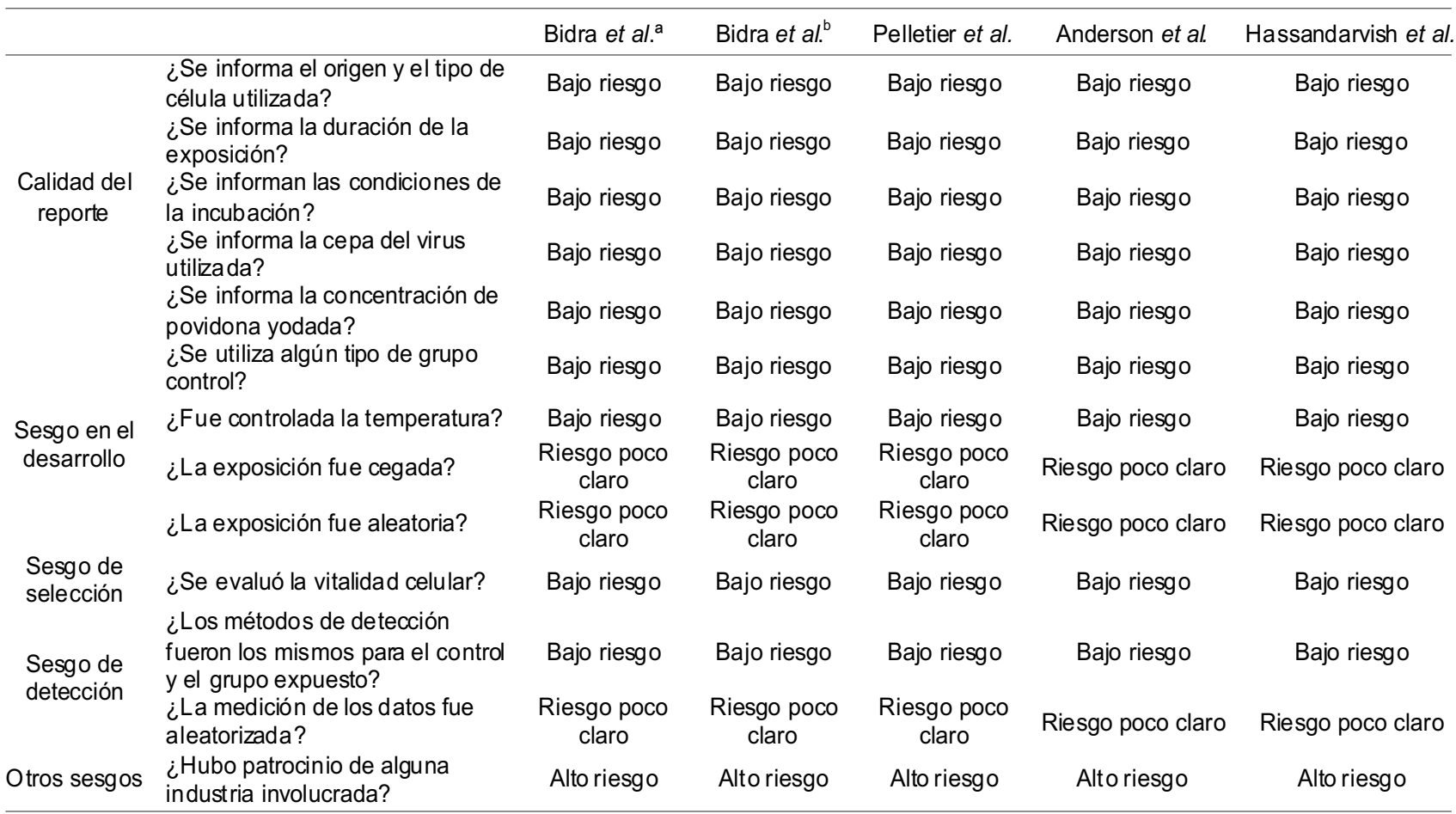

a Comparison of in vitro Inactivation of SARS CoV-2 with Hydrogen Peroxide and Povidone-lodine Oral Antiseptic Rinses.

${ }^{b}$ Rapid In-vitro inactivation of severe acute respiratory syndrome coronavirus 2 (SARS-COV-2) usiing povidone-iodine oral antiseptic rinse.

Tabla IV. Riesgo de sesgo en estudios in vivo $(\mathrm{n}=2)$

\begin{tabular}{llcc}
\hline \multicolumn{1}{c}{ Tipo de sesgo } & Fuente de sesgo & Mohamed et al. & Seneviratne et al. \\
\hline Sesgo de selección & Generación aleatoria de la secuencia & Bajo riesgo & Bajo riesgo \\
& Ocultamiento de la asignación & Alto riesgo & Riesgo poco claro \\
Sesgo de desarrollo & Cegamiento de participantes y personal & Alto riesgo & Riesgo poco claro \\
Sesgo de detección & Cegamiento de la evaluación de resultados & Riesgo poco claro & Riesgo poco claro \\
Sesgo de deserción & Datos de resultados incompletos & Bajo riesgo & Bajo riesgo \\
Sesgo de reporte & Reporte selectivo & Bajo riesgo & Bajo riesgo \\
Otros sesgos & Conflicto de interés, financiamiento del ensayo & Bajo riesgo & Bajo riesgo \\
\hline
\end{tabular}


Resultados de estudios in vitro. Se seleccionaron 5 estudios in vitro que evaluaron la actividad virucida de povidona yodada como colutorio contra medios celulares que presentaban distintas cepas del virus SARSCoV-2.

La efectividad de su uso fue evaluada a través de ensayos de dilución de punto final que midieron la reducción en la titulación viral y la reducción logarítmica en comparación al grupo control, para lo cual se utilizaron los métodos de Spearman Kärber y de Reed Muench. Todos los estudios presentaron algún tipo de grupo control negativo.
Tres de los estudios analizados utilizaron el método de Reed y Muench para estimar la titulación viral (Tabla V). En los tres ensayos, todas las concentraciones de povidona yodada demostraron tener un efecto virucida en los distintos tiempos de exposición, evidenciando una reducción en los títulos virales que alcanzó el valor mínimo de detección por el ensayo (0,67 Log10 CCID50/0,1 mL).

Dos trabajos utilizaron el método de Spearman Kärber para medir la titulación viral (Tabla VI). La eficacia virucida se estimó a partir de una adaptación de la norma EN14476 (European Committee for

Tabla V. Estudios in vitro que evaluaron la eficacia virucida de povidona yodada como colutorio contra el virus SARS-CoV2 utilizando el método de Reed y Muench.

\begin{tabular}{|c|c|c|c|c|c|c|c|}
\hline Autor & Publica ción & País & $\begin{array}{c}\text { Concentraciones } \\
\text { Utilizadas }\end{array}$ & $\begin{array}{l}\text { Tiempo de } \\
\text { contacto }\end{array}$ & $\begin{array}{c}\text { Titulación } \\
\text { viral }\end{array}$ & $\begin{array}{l}\text { Reducción } \\
\text { logarítmica }\end{array}$ & $\begin{array}{c}\text { Control } \\
\text { negativo }\end{array}$ \\
\hline \multirow{6}{*}{ Bidra et al. ${ }^{a}$} & \multirow{6}{*}{ Junio 2020} & \multirow{6}{*}{$\begin{array}{l}\text { Estados } \\
\text { Unidos }\end{array}$} & $0,5 \%$ & $15 \mathrm{~s}$ & $<0,67$ & $>4,33$ & \multirow{6}{*}{ Agua } \\
\hline & & & $0,5 \%$ & $30 \mathrm{~s}$ & $<0,67$ & $>3,63$ & \\
\hline & & & $1,25 \%$ & $15 \mathrm{~s}$ & $<0,67$ & $>4, .33$ & \\
\hline & & & $1,25 \%$ & $30 \mathrm{~s}$ & $<0,67$ & $>3,63$ & \\
\hline & & & $1,5 \%$ & $15 \mathrm{~s}$ & $<0,67$ & $>4,33$ & \\
\hline & & & $1,5 \%$ & $30 \mathrm{~s}$ & $<0,67$ & $>3,63$ & \\
\hline \multirow{6}{*}{ Bidra et al. ${ }^{\mathrm{b}}$} & \multirow{6}{*}{ Junio 2020} & \multirow{6}{*}{$\begin{array}{l}\text { Estados } \\
\text { Unidos }\end{array}$} & $0,5 \%$ & $15 \mathrm{~s}$ & $<0,67$ & 3,0 & \multirow{6}{*}{ Agua } \\
\hline & & & $0,5 \%$ & $30 \mathrm{~s}$ & $<0,67$ & 3,33 & \\
\hline & & & $0,75 \%$ & $15 \mathrm{~s}$ & $<0,67$ & 3,0 & \\
\hline & & & $0,75 \%$ & $30 \mathrm{~s}$ & $<0,67$ & 3,33 & \\
\hline & & & $1,5 \%$ & $15 \mathrm{~s}$ & $<0,67$ & 3,0 & \\
\hline & & & $1,5 \%$ & $30 \mathrm{~s}$ & $<0,67$ & 3,33 & \\
\hline \multirow{3}{*}{ Pelletier et al. } & \multirow{3}{*}{ Septiembre 2020} & \multirow{3}{*}{$\begin{array}{l}\text { Estados } \\
\text { Unidos }\end{array}$} & $0,5 \%$ & & $<0,67$ & 4,63 & \multirow{3}{*}{ Agua } \\
\hline & & & $0,75 \%$ & $60 \mathrm{~s}$ & $<0,67$ & 4,63 & \\
\hline & & & $1,5 \%$ & & $<0,67$ & 4,63 & \\
\hline
\end{tabular}

aComparison of In vitro Inactivation of SARS CoV-2 with Hydrogen Peroxide and Povidone-lodine Oral Antiseptic Rinses.

${ }^{b}$ Rapid In-vitro inactivation of severe acute respiratory syndrome coronavirus 2 (SARS-COV-2) usiing povidone-iodine oral antiseptic rinse. La titulación viral se mide como el Log10 CCID50 de virus por $0.1 \mathrm{~mL}$. El límite inferior de detección en los ensayos corresponde a 0,67 Log10 CCID50 por $0.1 \mathrm{~mL}$. La reducción logarítmica equivale a la reducción de la titulación viral en comparación con el grupo control.

Tabla VI. Estudios in vitro que evaluaron la eficacia virucida de povidona yodada como colutorio contra el virus SARS-CoV2 utilizando el método de Spearman Kärber.

\begin{tabular}{|c|c|c|c|c|c|c|}
\hline Autor & Publicación & País & $\begin{array}{c}\text { Concentraciones } \\
\text { Utilizadas }\end{array}$ & $\begin{array}{c}\text { Tiempos } \\
\text { utilizados }\end{array}$ & $\begin{array}{l}\text { Reducción } \\
\text { logarítmica }\end{array}$ & Control negativo \\
\hline \multirow{7}{*}{ Anderson et al. } & \multirow{7}{*}{ Julio 2020} & \multirow{7}{*}{ Singapur } & $0,5 \%$ & & $\geq 4,00$ & \multirow{7}{*}{$\begin{array}{l}\text { Buffer fosfato } \\
\text { salino }\end{array}$} \\
\hline & & & $1 \%$ & $30 \mathrm{~s}$ & $\geq 4,00$ & \\
\hline & & & 0,5 \% (Limpio) & $15 \mathrm{~s}$ & $>4,00$ & \\
\hline & & & 0,5 \% (Limpio) & $30 \mathrm{~s}$ & $>5,00$ & \\
\hline & & & 0,5 \% (Limpio) & $60 \mathrm{~s}$ & $>5,00$ & \\
\hline & & & $0,5 \%$ (Sucio) & $15 \mathrm{~s}$ & $>4,00$ & \\
\hline & & & $0,5 \%$ (Sucio) & $30 \mathrm{~s}$ & $>5,00$ & \\
\hline \multirow{7}{*}{$\begin{array}{l}\text { Hassandarvish et } \\
\text { al. }\end{array}$} & \multirow{7}{*}{$\begin{array}{c}\text { Diciembre } \\
2020\end{array}$} & \multirow{7}{*}{ Malasia } & 0,5\% (Sucio) & $60 \mathrm{~s}$ & $>5,00$ & \multirow{7}{*}{ Agua destilada } \\
\hline & & & $1 \%$ (Limpio) & $15 \mathrm{~s}$ & $>5,00$ & \\
\hline & & & $1 \%$ (Limpio) & $30 \mathrm{~s}$ & $>5,00$ & \\
\hline & & & $1 \%$ (Limpio) & $60 \mathrm{~s}$ & $>5,00$ & \\
\hline & & & $1 \%$ (Sucio) & $15 \mathrm{~s}$ & $>5,00$ & \\
\hline & & & $1 \%$ (Sucio) & $30 \mathrm{~s}$ & $>5,00$ & \\
\hline & & & $1 \%$ (Sucio) & $60 \mathrm{~s}$ & $>5,00$ & \\
\hline
\end{tabular}


Standardization, 2013), donde se considera que el antiséptico posee una actividad virucida eficaz cuando la reducción en la titulación viral es mayor o igual a $4 \log 10(99,99 \%)$. En ambos trabajos, todas las concentraciones de povidona yodada utilizadas evidenciaron una actividad virucida eficaz en los distintos tiempos establecidos.

Resultados de estudios In vivo. Se seleccionaron 2 estudios in vivo que evaluaron la efectividad de la povidona yodada como colutorio en la disminución de la carga viral de pacientes con COVID-19, los cuales reclutaron pacientes diagnosticados mediante RTPCR. Las mediciones de la carga viral se realizaron de manera previa al enjuague y en distintos tiempos posteriores a este. Ambos estudios contaron con algún tipo de grupo control (enjuague con agua y/o no intervención).

Seneviratne et al. midieron el efecto del antiséptico empleando una solución al 0,5\% compuesta por $5 \mathrm{ml}$ de povidona yodada y $5 \mathrm{ml}$ de agua estéril, con un tiempo de enjuague de 30 segundos. Se incluyeron 6 pacientes con diagnóstico positivo para SARSCoV-2 mediante RT-PCR obtenido por hisopado nasofaríngeo. La carga viral en los pacientes fue evaluada mediante una muestra salival de manera previa al enjuague, a los 5 minutos, y a las 3 y 6 horas posteriores a este. Para la evaluación de los resultados se analizó el valor Ct que corresponde al número de ciclos de amplificación necesarios para que el gen en estudio supere un nivel de umbral. Esto se correlaciona inversamente con la carga viral y puede proporcionar un método indirecto de cuantificación del número de copias de ARN viral en la muestra (Rao et al., 2020). Si bien hubo una variación en todos los momentos posteriores al enjuague con povidona yodada comparado con el grupo control (agua), sólo fue estadísticamente significativa en la medición de las 6 horas posterior a su aplicación. Sin embargo, los da-

Tabla VII. Comparación de resultados de RT-PCR de COVID-19 del grupo PI versus grupo control.

\begin{tabular}{ccccc}
\hline Día & Resultado PCR & PI 1\% $15 \mathrm{ml}, \mathrm{n}$ & $\begin{array}{c}\text { Grupo } \\
\text { control }\end{array}$ & $\begin{array}{c}\text { Agua potable 100 } \\
\mathrm{ml}\end{array}$ \\
\hline \multirow{4}{*}{4} & Negativo & 5 & 1 & 2 \\
& Positivo & 0 & 2 & 3 \\
& Indeterminado & 0 & 2 & 0 \\
6 & Negativo & 5 & 0 & 2 \\
6 & Positivo & 0 & 3 & 1 \\
& Indeterminado & 0 & 2 & 2 \\
12 & Negativo & 5 & 1 & 2 \\
& Positivo & 0 & 3 & 2 \\
\hline
\end{tabular}

tos con los que se obtuvieron estos resultados no estaban disponibles en la publicación. Se intentó contactar al autor para obtener dicha información sin recibir respuesta.

Mohamed et al., comparó el efecto del uso de colutorios de povidona yodada, agua y la ausencia de intervención sobre la carga viral de pacientes COVID19. Para el colutorio, se utilizó una solución de $10 \mathrm{ml}$ de povidona yodada al $1 \%$ con un tiempo de enjuague de 30 segundos. El agua utilizada fue obtenida en el grifo y para el enjuague se utilizaron $100 \mathrm{ml}$ durante 30 segundos. Se incluyó a pacientes mayores de 18 años, asintomáticos, y con menos de 5 días transcurridos desde el diagnóstico. Los 10 pacientes seleccionados realizaron enjuagues durante 7 días, tres veces al día. Se realizó toma de muestra oro y nasofaríngea antes del primer enjuague en los días 4 , 6 y 12. Se consideró resultado positivo de RT-PCR si ambas muestras obtenían un Ct igual o menor a 45, negativo si no se obtenía un $\mathrm{Ct}$ e indeterminado si había discordancia entre los dos hisopados. Los resultados obtenidos se muestran en la Tabla VII.

En el grupo que utilizó povidona yodada, todos los participantes obtuvieron resultados de RT-PCR negativos en los tres días evaluados, Por su parte, el grupo sin intervención obtuvo mayoritariamente resultados positivos o indeterminados en todos los días evaluados. No se reportaron efectos adversos frente al uso de povidona yodada.

\section{DISCUSIÓN}

La dosis profiláctica de povidona yodada que ha recomendado la Asociación Dental Americana (ADA) para minimizar el riesgo de transmisión del SARS-CoV-2 durante la consulta dental corresponde a una solución del $0,2 \%$, pero sin evidencia que la respalde (American Dental Association). La mínima concentración del colutorio que ha resultado efectiva en los estudios in vitro evaluados corresponde a $0,5 \%$, cuya aplicación se recomienda por al menos 15 segundos. Esto corresponde a un tiempo de enjuague fácilmente realizable en la práctica clínica, y resulta similar al tiempo mínimo necesario para inactivar los virus SARS-CoV y MERS-CoV (Eggers et al.). De igual forma, el tiempo mínimo utilizado en los trabajos in vivo analizados 
en esta revisión correspondió a 15 segundos, sin embargo, estos no obtuvieron resultados definitorios.

Los resultados de los trabajos in vivo no resultan comparables debido a la gran heterogeneidad que existe en su metodología. Lo anterior supone una limitación en esta revisión. Además, uno de los ensayos no presenta toda la información necesaria para respaldar sus resultados, lo que resulta una amenaza para la validez de sus conclusiones (Seneviratne et al.). Sumado a esto, ambos trabajos poseen tamaños muestrales pequeños y se requieren de grandes ensayos controlados aleatorizados para evaluar de mejor manera este tipo de intervenciones (Silverman, 2009).

En los trabajos in vitro, la mayor limitación corresponde al riesgo de sesgo producido por los conflictos de intereses financieros que genera el patrocinio recibido por industrias farmacéuticas, lo cual resulta sumamente relevante si se considera que las investigaciones con algún tipo de patrocinio económico tienen un mayor riesgo relativo $(R R=1.98)$ de presentar conclusiones favorables en comparación a aquellos sin financiamiento (Hansen et al., 2019).

Se debe considerar que el uso de povidona yodada como colutorio debe valorarse acorde a las comorbilidades de cada paciente. Su aplicación debe evitarse en mujeres embarazadas, madres lactantes y personas con enfermedad tiroidea activa (Ader et al., 1988; Nobukuni \& Kawahara, 2002). Del mismo modo, no se recomienda su uso en personas con el antecedente de reacción anafiláctica posterior a su aplicación (Gray et al., 2013).

En conclusión, los resultados de la presente revisión indican que el uso profiláctico de povidona yodada como colutorio contra el virus SARS-CoV-2 es respaldado por los resultados de distintos trabajos in vitro, pero se requiere de un mayor número de ensayos clínicos para evaluar su efectividad en la atención dental. Los estudios in vivo que formaron parte de esta revisión presentan una gran heterogeneidad y un pequeño número de participantes, por lo que sus resultados deben ser interpretados con precaución.

\section{AGRADECIMIENTOS}

Agradecimientos al Dr. Pouya Hassandarvish por responder a nuestras dudas sobre las cepas utilizadas en su investigación.
ANWANDTER, A.; LEFNO-DIOCARES. C.; VALERIA, C. \& ROBLEDO, D. Povidone iodine as an oral antiseptic in the reduction of SARS-CoV-2 viral load: Literature review. Int. J. Odontostomat., 15(4):997-1004, 2021.

ABSTRACT: It has been suggested that the use of oral antiseptics could reduce the viral load of SARS-CoV-2 virus in patients during dental care, but without evidence to support its effectiveness. The objective of this study was to determine the effectiveness of povidone iodine mouthwash in reducing the viral load of SARS-CoV-2 virus. A literature search was conducted in PubMed, Biblioteca Virtual enSalud, SciELO, Web of Science and EBSCOhost. Clinical studies in patients with COVID-19 or in vitro studies with SARS-CoV-2 strains that used povidone-iodine mouthwash as a form of intervention, published between January 2019 and January 2021, were included. The selection of articles was carried out in two phases by two authors independently. After removing duplicate articles, 53 references were kept. Finally, 2 in vivo studies and 5 in vitro studies were included for the qualitative review. In the in vitro studies, all concentrations of povidone iodine showed effective virucidal activity at the different exposure times, where the minimum effective concentration corresponded to $0.5 \%$ in 15 seconds. In vivo studies showed positive results towards the use of povidone iodine, but with small sample sizes and great heterogeneity in their methodology. The prophylactic use of povidone iodine mouthwash against the SARS-CoV2 virus is supported by in vitro studies, with application times easily achievable in dental care, but a large number of randomized controlled trials are required to verify its effectiveness in clinical practice.

KEY WORDS: COVID-19, povidone-iodine, mouthwashes, dental prophylaxis.

\section{REFERENCIAS BIBLIOGRÁFICAS}

Ader, A.; Paul, T.; Reinhardt, W.; Safran, M.; Pino, S.; McArthur, W. \& Braverman, L. Effect of mouth rinsing with two polyvinylpyrrolidone-iodine mixtures on iodine absorption and thyroid function. J. Clin. Endocrinol. Metab., 66(3):632-5, 1988.

American Dental Association. ADA Interim Guidance for Minimizing Risk of COVID-19 Transmission. Chicago, American Dental Association, 2020.

Anderson, D.; Sivalingam, V.; Kang, A.; Ananthanarayanan, A.; Arumugam, H.; Jenkins, T.; Hadjiat, Y. \& Eggers, M. Povidoneiodine demonstrates rapid in vitro virucidal activity against SARSCoV-2, the virus causing COVID-19 disease. Infect. Dis. Ther., 9(3):669-75, 2020.

Bidra, A.; Pelletier, J.; Westover, J.; Frank, S.; Brown, S. \& Tessema, B. Comparison of In vitro Inactivation of SARS CoV-2 with Hydrogen Peroxide and Povidone-lodine Oral Antiseptic Rinses. J. Prosthodont., 29(7):599-603, 2020a.

Bidra, A.; Pelletier, J.; Westover, J.; Frank, S.; Brown, S. \& Tessema, B. Rapid in-vitro inactivation of Severe Acute Respiratory Syndrome Coronavirus 2 (SARS-CoV-2) using povidone-iodine oral antiseptic rinse. J. Prosthodont., 29(6):529-33, 2020b. 
Eggers, M.; Koburger-Janssen, T.; Eickmann, M. \& Zorn, J. In vitro bactericidal and virucidal efficacy of povidone-iodine gargle/ mouthwash against respiratory and oral tract pathogens. Infect. Dis. Ther., 7(2):249-59, 2018.

European Committee for Standardization. Chemical disinfectants and antiseptics - Quantitative suspension test for the evaluation of virucidal activity in the medical area - Test method and requirements (Phase 2/Step 1). European Standard, 2013.

Frank, S.; Capriotti, J.; Brown, S. \& Tessema, B. Povidone-iodine use in sinonasal and oral cavities: a review of safety in the COVID19 era. Ear Nose Throat J., 99(9):586-93, 2020.

Golbach, L.; Portelli, L.; Savelkoul, H.; Terwel, S.; Kuster, N.; de Vries, R. \& Verburg-van Kemenade, B. Calcium homeostasis and low-frequency magnetic and electric field exposure: A systematic review and meta-analysis of in vitro studies. Environ. Int., 92-93:695-706, 2016.

Gray, P.; Katelaris, C. \& Lipson, D. Recurrent anaphylaxis caused by topical povidone-iodine (Betadine). J. Paediatr. Child. Health, 49(6):506-7, 2013.

Hansen, C.; Lundh, A.; Rasmussen, K. \& Hróbjartsson, A. Financial conflicts of interest in systematic reviews: Associations with results, conclusions, and methodological quality. Cochrane Database Syst. Rev., 8(8):MR000047, 2019.

Harrel, S. \& Molinari, J. Aerosols and splatter in dentistry: A brief review of the literature and infection control implications. J. Am. Dent. Assoc., 135(4):429-37, 2004.

Hassandarvish, P.; Tiong, V.; Mohamed, N.; Arumugam, H.; Ananthanarayanan, A.; Qasuri, M.; Hadjiat, Y. \& Abubakar, S. In vitro virucidal activity of povidone iodine gargle and mouthwash against SARS-CoV-2: implications for dental practice. Br. Dent. J., 2020. DOI: https://www.doi.org/10.1038/s41415-020-2402-0

Higgins, J.; Altman, D.; Gøtzsche, P.; Jüni, P.; Moher, D.; Oxman, A.; Savovic', J.; Schulz, K.; Weeks, L. \& Sterne, J. The Cochrane Collaboration's tool for assessing risk of bias in randomised trials. BMJ, 343:d5928, 2011.

Huang, C.; Wang, Y.; Li, X.; Ren, L.; Zhao, J.; Hu, Y.; Zhang, L.; Fan, G.; Xu, J.; Gu, X.; et al. Clinical features of patients infected with 2019 novel coronavirus in Wuhan, China. Lancet, 395(10223):497-506, 2020.

Kramer, A.; Dissemond, J.; Kim, S.; Willy, C.; Mayer, D.; Papke, R.; Tuchmann, F. \& Assadian, O. Consensus on Wound Antisepsis: Update 2018. Skin Pharmacol. Physiol., 31(1):28-58, 2018.

Mohamed, N.; Baharom, N.; Wan Sulaiman, W.; Rashid, Z.; Ken, W.; Ali, U.; Othman, S.; Samat, M.; Kori, N.; Periyasamy, P.; et al. Earlyviral Clearance among COVID-19 patients when gargling with povidone-iodine and essential oils: A clinical trial. Int. Med. J., 27(6):651-4, 2020.

Nobukuni, K. \& Kawahara, S. Thyroid function in nurses: The influence of povidone-iodine hand washing and gargling. Dermatology, 204(1):99-102, 2002.

O'Donnell, V. B.; Thomas, D.; Stanton, R.; Maillard, J.; Murphy, R.; Jones, S.; Humphreys, I., Wakelam, M.; Fegan, C.; Wise, M.; et al. Potential Role of Oral Rinses Targeting the Viral Lipid Envelope in SARS-CoV-2 Infection. Function (Oxf.), 1(1):zqaa002, 2020.

Organización Mundial de la Salud (OMS). Alocución de apertura del Director General de la OMS en la rueda de prensa sobre la COVID-19 celebrada el 11 de marzo de 2020. Ginebra, Organización Mundial de la Salud, 2020b.

Organización Mundial de la Salud (OMS). Los nombres de la enfermedad por coronavirus (COVID-19) y del virus que la causa. Ginebra, Organización Mundial de la Salud, 2020a.

Pelletier, J.; Tessema, B.; Frank, S.: Westover, J.; Brown, S. \& Capriotti, J. Efficacy of povidone-iodine nasal and oral antiseptic preparations against Severe Acute Respiratory SyndromeCoronavirus 2 (SARS-CoV-2). Ear Nose Throat J., 100(2):1926, 2020.
Rao, S.; Manissero, D.; Steele, V. \& Pareja, J. A Narrative Systematic Review of the Clinical Utility of Cycle Threshold Values in the Context of COVID-19. Infect. Dis. Ther., 9(3):573-86, 2020.

Seneviratne, C.; Balan, P.; Ko, K.; Udawatte, N.; Lai, D.; Ng, D.; Venkatachalam, I.; Lim, K.; Ling, M.; Oon, L.; et al. Efficacy of commercial mouth-rinses on SARS-CoV-2 viral load in saliva: randomized control trial in Singapore. Infection, 49(2):305-11, 2020.

Silverman, S. From randomized controlled trials to observational studies. Am. J. Med., 122(2):114-20, 2009.

To, K.; Tsang, O.; Yip, C.; Chan, K.; Wu, T.; Chan, J.; Leung, W.; Chik, T.; Choi, C.; Kandamby, D.; et al. Consistent Detection of 2019 Novel Coronavirus in Saliva. Clin. Infect. Dis., 71(15):841 3, 2020.

Wiersinga, W.; Rhodes, A.; Cheng, A.; Peacock, S. \& Prescott, H. Pathophysiology, transmission, diagnosis, and treatment of Coronavirus Disease 2019 (COVID-19): A Review. JAMA, 324(8):782-93, 2020.

Dirección para correspondencia:

Cristóbal Román Lefno Diocares

Bosques de Entre Ríos

Pasaje Elena 3 \#260

Valdivia

Región de los Ríos

CHILE

E-mail: lefno@hotmail.com 